\begin{tabular}{|c|l|}
\hline Title & Molecular chirality induction to an achiral $\pi$-conjugated polymer by circularly polarized light \\
\hline Author(s) & Wang, Y ue; Sakamoto, Takeshi; Nakano, Tamaki \\
\hline Citation & $\begin{array}{l}\text { Chemical Communications, 48(13), 1871-1873 } \\
\text { https:/doi.org/10.1039/c2c17027b }\end{array}$ \\
\hline Issue Date & 2012-02-11 \\
\hline Doc URL & http://hdl.handle.net/2115/50988 \\
\hline Rights & Chem. Commun., 2012, 48, 1871-1873- Reproduced by permission of The Royal Society of Chemistry (RSC) \\
\hline Type & article (author version) \\
\hline File Information & Cc48_13_1871-1873.pdf \\
\hline
\end{tabular}

Instructions for use 


\title{
Molecular Chirality Induction to an Achiral $\pi$-Conjugated Polymer by Circularly Polarized Light **
}

\author{
Yue Wang, ${ }^{a}$ Takeshi Sakamoto, ${ }^{b}$ and Tamaki Nakano* ${ }^{b}$ \\ Received (in $X X X, X X X)$ Xth $X X X X X X X X X 200 X$, Accepted Xth $X X X X X X X X X 200 X$ \\ ${ }_{5}$ First published on the web Xth $X X X X X X X X X 200 X$ \\ DOI: 10.1039/b000000x
}

\begin{abstract}
A preferred-handed helical conformation was induced to poly(9.9-dioctylfluren-2,7-diyl) (PDOF) in a thin film form upon irradiation with single-handed circularly polarized light (CPL) where the induction was reversible.
\end{abstract}

10

Chiral polymers create important materials exhibiting valuable functions. Polymers with helical chirality, in particular, are known to feature excellent functions, including chiral recognition ability. ${ }^{1,2}$ Optically active helical polymers

15 have been synthesized by asymmetric polymerization using complexes of chiral ligands and metallic species or by supramolecular interactions between polymer chains and external chiral molecules. $^{3-5}$ Herein, we report a novel method of preparation of an optically active artificial polymer 20 with molecular chirality from an achiral source material using single-handed circularly polarized light (CPL).

The potential for use of CPL for the production of optically active materials was recognized as early as the 19th century, ${ }^{6,7}$ and there have been examples of CPL-assisted asymmetric 25 syntheses. ${ }^{8,9}$ There has, however, been no clear example of turning an optically inactive, achiral polymer with no specific conformation into an optically active form with a preferredhanded helical strcture. In this work, we have succeeded in preparing optically active poly(9,9-di-n-octylfluoren-2,7-diyl) 30 (PDOF) in thin film using CPL as the only source of chirality.

PDOF has been applied as a light-emitting and holetransport material for organic light-emitting diodes (OLEDs). ${ }^{10,11}$ This polymer has no center of chirality. Although axial chirality around single bonds connecting 35 fluorene units is possible, due to the extremely fast conformational dynamics, this polymer can virtually be regarded achiral in solution. In the solid state, where conformational dynamics are retarded, however, exchange between $\mathrm{P}$ - and M-twists around single bonds may be 40 remarkably slow, and enrichment of either of the mirror image twists may be conducted by external stimuli. PDOF in the solid state consists of chiral twisted sequences and planarzigzag or $2_{1}$ helical sequences forming the achiral $\beta$-phase. ${ }^{12}$

${ }^{a}$ Graduate School of Chemistry and Chemical Sciences, Hokkaido 45 University, Sapporo 060-8628, Japan.

${ }^{b}$ Division of Biotechnology and Macromolecular Chemistry, Faculty of Engineering, Hokkaido University, Sapporo 060-8628, Japan. E-mail: nakanot@eng.hokudai.ac.jp; Fax: +81 117066869

$\uparrow$ This communication is dedicated to Professor Dr Tatsuki Kitayama on 50 the occasion of his 60th birthday ("Kanreki").

\$Electronic Supplementary Information (ESI) available: Experimental details, ${ }^{1} \mathrm{H}$ NMR, UV, CD, FT-IR spectra, SEC curves, DSC charts, XRD profiles, and polarized optical micrographs of the film sample.
Based on this concept, PDOF was irradiated with CPL in a ${ }_{55}$ thin-film form under $\mathrm{N}_{2}$ atmosphere. Film samples were prepared by a solution cast method (thickness ca. $0.05 \mu \mathrm{m}$ ).

Intense bisignate Cotton effects due to a $\pi-\pi^{*}$ transition were observed in circular dichroism (CD) spectra for a PDOF film upon CPL irradiation (Fig. 1). Upon irradiation using a ${ }_{60} 500-\mathrm{W} \mathrm{Hg}$-Xe lamp-based R-CPL for $6 \mathrm{~min}$ at $23^{\circ} \mathrm{C}$, a clear negative Cotton band emerged at around $400 \mathrm{~nm}$ (Fig. 1 CD1). This band almost completely disappeared upon L-CPL irradiation for $6 \mathrm{~min}$ (Fig. $1 \mathrm{CD}-2)$. Further irradiation with L-CPL for an additional $6 \mathrm{~min}$ (total duration = $12 \mathrm{~min}$ ) gave ${ }_{65}$ rise to a positive $\mathrm{CD}$ band at around $400 \mathrm{~nm}$ (Fig. $1 \mathrm{CD}-3$ ). The first and last spectra were almost symmetrical. Thus, clear chiral induction and chiral switching were attained for PDOF using CPL. Similar chiral induction was also attained by sunlight (ESI: Fig. S1).

70
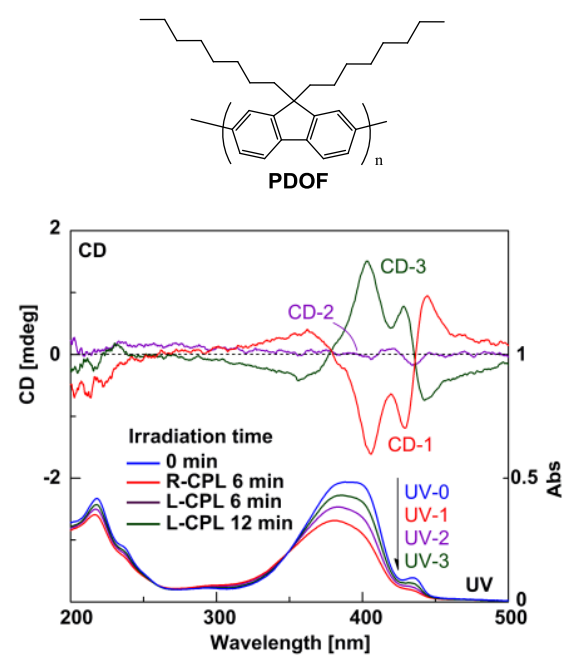

Fig. 1. CD and UV spectral changes of PDOF in film on irradiation with R-CPL for 0 min (blue, UV-0) and 6 min (red, UV-1 and CD-1) 75 and on irradiation with L-CPL for 6 min (purple, UV-2 and CD-2) and for additional $12 \mathrm{~min}$ (green, UV-3 and CD-3). CD spectra were obtained by averaging those recorded at different film orientations (angles) with the film face positioned vertically to the incident light beam for measurement.

80

The CD spectral patterns are closely similar to those reported for optically active polyfluorenes bearing chiral sidechain groups with a preferred-handed twisted, helical conformation. $^{13,14}$ The CD spectra shown in Fig. 1 85 consequently indicate that PDOF irradiated with CPL has a bias of $\mathrm{P}$ - or M-twist and that the entire polymer chain is in a preferred-handed helical conformation. Although the $\mathrm{CD}$ patterns observed in this study were not completely indentical 
to those in literature, ${ }^{13,14}$ the slight discrepancies may be based on differences in helical pitch. The relation between the absolute configuration of the helix and the sign of the CD spectra has been theoretically investigated: for a model trimer 5 of fluorene, a positive Cotton effect at $410 \mathrm{~nm}$ due to the $\mathrm{S}_{0^{-}}$ $\mathrm{S}_{1}$ transition has been predicted to be based on an M-twist. ${ }^{13 \mathrm{~b}}$ Hence, L-CPL induces an M-helical sense for PDOF.

When the irradiated polymer was dissolved in tetrahydrofuran, it did not indicate Cotton effects anymore. 10 This indicates that the induced conformation is stable only in the solid state. In addition, racemization in the solid state also took place on heating film at $160^{\circ} \mathrm{C}$ (ESI: Fig. S2).

a

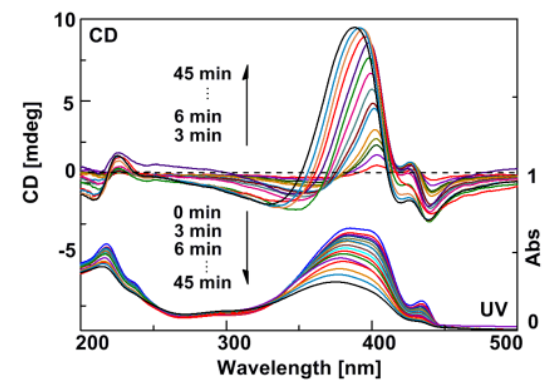

b
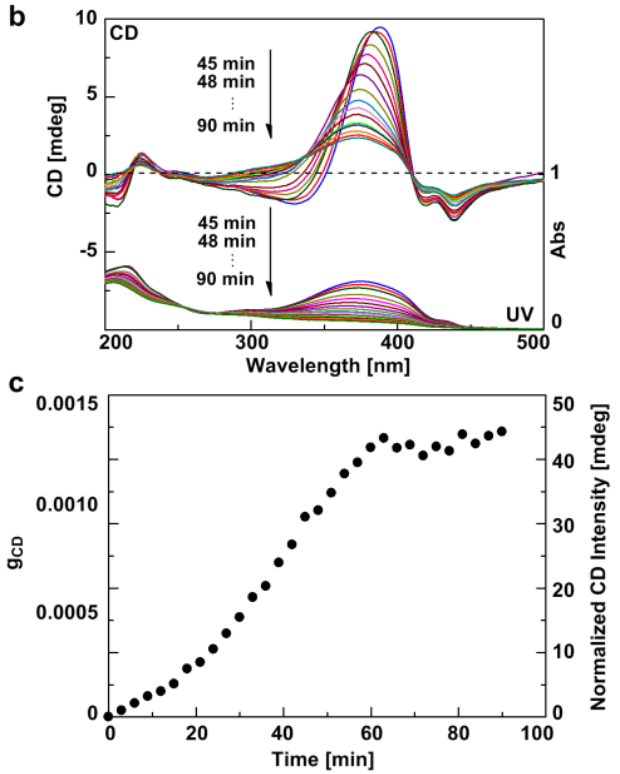

Fig. 2. CD and UV spectra of PDOF measured every 3 min during L15 CPL irradiation for a $0-45$ min duration at ambient temperature (22$23^{\circ} \mathrm{C}$ ) (a), those measured every 3 min during L-CPL irradiation for a 45-90 min duration at ambient temperature $\left(22-23^{\circ} \mathrm{C}\right)(\mathrm{b})$, and $\mathrm{g}_{\mathrm{CD}}$ (normalized CD intensity)-vs.-irradiation time plot based on the data from $\mathbf{a}$ and $\mathbf{b}(\mathrm{c})$. $\mathrm{g}_{\mathrm{CD}}$ in $\mathbf{c}$ was estimated for the CD signal at around $20380 \mathrm{~nm}$. CD intensity in c was normalized for 1.0-UV absorbance.

The intensity of the induced CD spectra varied with the irradiation time with L-CPL in extended irradiation durations (Fig. 2a and b). The apparent CD intensity first increased and 25 then decreased with the irradiation time, and, at the same time, the UV absorption intensity decreased and slightly blue shifted continuously with the irradiation time. The anisotropy factor $\left(g_{\mathrm{CD}}\right)$ increased with the irradiation time and eventually leveled off after ca. 60-min irradiation (Fig. 2c). The 30 maximum $\mathrm{g}_{\mathrm{CD}}$ was $0.0013\left(44 \mathrm{~m}^{\circ}\right.$ for 1.0 absorbance at 374 $\mathrm{nm})$ after 90-min irradiation.
The decrease in intensity and blue shifts in UV spectra are ascribed in part to the hypochromic effect due to interchain $\pi-\pi$ stacking in an H-type aggregation form. $\pi$-Stacking 35 leads to a pronounced hypochromicity, ${ }^{15}$ and $\mathrm{H}$-aggregation results in blue shifts. ${ }^{16}$ This assumption is supported by the fluorescent spectra. While the PDOF film before irradiation indicated emission bands peaking around $440 \mathrm{~nm}, 470 \mathrm{~nm}$, and $500 \mathrm{~nm}$, the film after L-CPL irradiation for $12 \mathrm{~min}$ at ${ }_{40} 23^{\circ} \mathrm{C}$ indicated a broad emission band peaking at around 530 $\mathrm{nm}$ in addition to the aforementioned bands (ESI: Fig. S3). The broad emission is ascribed mainly to excimer emission and partially to oxidized units (vide infra). Increased conformational homogeneity induced by CPL may possibly 45 have made close interchain interaction more feasible, although the interaction seems to be limited to relatively short-distance ranges, as no apparent liquid crystallinity was confirmed for the irradiated film sample.

In addition, the decrease in intensity of the UV band at 50 around $435 \mathrm{~nm}$ due to the $\beta$-phase seems more obvious than at around $400 \mathrm{~nm}$, suggesting that the molecular motion of twisted sequences on excitation and deactivation (twistcoplanar transition, vide infra) induces conformational transition of planar-zigzag sequences contained in the $\beta$-phase 55 in the vicinity into twisted forms by steric interactions.

Effect of CPL on chemical structure of PDOF was investigated by means of FT-IR, ${ }^{1} \mathrm{H}$ NMR, and SEC analyses (ESI: Fig. S4-S6). Although the apparent molecular weight estimated by SEC was almost unchanged before and after 60 irradiation, unabmigously indicating that no chain session took place, FT-IR spectra suggested the formation of fluorenone units (keto defects). ${ }^{17 a}$ The amount of fluorenone units was asummed to be only ca. $0.3 \%$ and ca. $0.5 \%$ after irradiation for $6 \mathrm{~min}$ and $90 \mathrm{~min}$, respectively, based on ${ }^{1} \mathrm{H}$ ${ }_{65}$ NMR spectra. ${ }^{17 \mathrm{~b}}$ Therefore, they would not significantly contribute to the observed CD spectra. Whether the formation of a small amount of fluorenone units is correlated to chirality induction is not yet clear at this stage.

Although chirality induction mechanism is not completely 70 clear yet, it is proposed to be as follows. Biaryl compounds are known to undergo a conformational transition on photo excitation, where a twist conformation in the ground state is transformed into a coplanar form in excited states. ${ }^{18}$ This transition would be plausible for the fluorene-fluorene units in 75 PDOF. When P- and M-twists have different molar absorptivities toward single-handed CPL, either the P- or Mtwist will be preferentially excited. The excited enantiomeric twist will form $\mathrm{P}$ - and M-twists, resulting in an excess concentration of a twist with less likelihood of excitation by 80 the single-handed CPL used for irradiation (a reversible photo racemization process ${ }^{9}$ ). This mechasnism is supported by the facts that chemical structural change of PDOF was only slight and that the chiral induction was reversible.

The proposed mechanism of chiral induction is partially 85 supported by the facts that intensity of the induced CD spectra largely decreased on linearly polarized light (LPL) irradiation (ESI: Fig. S7). These results suggest that LPL, racemic CPL, induces racemization of the induced preferred-handed helix.

Achievable extent of chiral induction is related to the 
difference in molar absorptivity toward single-handed CPL between enantiomers. However, it is generally subtle for organic molecules with a $\pi-\pi^{*}$ transition. ${ }^{9 a}$ The difference is evaluated by Kuhn's $g_{\gamma}$ value $^{19}$ which is on the order of less 5 than $10^{-3}$ for typical aromatic organic molecules. Hence, the enantiomeric excess achievable by CPL in the photostationary state $\left(\gamma_{\text {PSS }}=1 / 2 \mathrm{~g}_{\gamma}\right)$ is extremely small, and the chiroptical properties of reversible photo racemization products are often negligible.

10 Although the $\mathrm{g}_{\gamma}$ of PDOF is unknown because the polymer is usually recognized as an achiral compound and its optically pure form is therefore unavailable, the intense $\mathrm{CD}$ may suggest a significant extent of chiral induction. The CD intensity of $44 \mathrm{~m}^{\circ}$ for 1.0-UV absorbance achieved by 90 -min ${ }_{15} \mathrm{CPL}$ irradiation is comparable to that of optically pure poly(9,9-bis( $(S)$-5-methylheptyl)fluoren-2,7-diyl) with chiral side-chain groups (ca. $50 \mathrm{~m}^{\circ}$ at $400 \mathrm{~nm}$ ), and it is ca. $1 / 45$ that of poly(9,9-bis((3S)-3,7-dimethyloctyl)fluoren-2,7-diyl). ${ }^{13 \mathrm{~b}}$

The significant chiral induction observed in this study may 20 be due in part to an amplification effect ${ }^{20}$ in the solid state. In the solid state, deactivation of an excited twist may not be a complete racemization process but, rather, an enriching process, where the chances of obtaining P-twist and M-twist from a coplanar excited chromophore are unequal due to the 25 steric effects of single-handed twists in the vicinity that have been formed previously in earlier excitation-deactivation events. This effect should be more obvious when the difference in the populations of $\mathrm{P}$ - and M-twists is larger, bringing about an apparent amplification. This proposal is 30 supported by the plots of effective CD intensity against irradiation time in Fig. 2c, where the slope of the curve tends to increase with increases in irradiation time. This is especially obvious in the early stages of irradiation, where levelling-off of the plots does not hide the increase in slope.

35 The PDOF film samples studied in this work seemed to be mostly glassy as supported by polarized-microscopic observations, thermal analyses, and X-ray diffraction experiments (ESI: Fig. S8-S10). Neither polarizedmicroscopic observations of film samples before nor after 40 irradiation at ambient temperature indicated a clear texture that might be related to crystalline or LC phases. In differential scanning calorimetric (DSC) analyses, moreover, films before and after irradiation indicated exothermic and endothermic responses at around $100-120^{\circ} \mathrm{C}$ and $130-150^{\circ} \mathrm{C}$, 45 respectively. These behaviors are typical of glassy, amorphous PDOF. ${ }^{21}$ Further, X-ray diffraction profiles before and after irradiation did not show responses due to crystalline domains. Hence, chirality induction by CPL under the conditions employed in this study took place mainly in an 50 amorphous solid state, and it mainly caused molecular conformational chirality.

In summary, we have successfully induced chirality to PDOF by CPL obtained from an artificial light source and the sun. This is the first report on preferred handed-helical 55 conformation induction to a virtually achiral main-chain conjugated polymer without the use of chemical substances but with only the aid of light. Polymer chirality induction by CPL has been demonstrated for a helical polyisocyanate bearing CPL-sensitive side-chain groups. ${ }^{22}$ In addition, CPL${ }_{60}$ induced chirality to film samples of vinyl polymers bearing photochromic azo moieties has been reported where observed chiroptical properties are assigned to formation of chiral liquid crystalline phases. $^{23}$ Our work on PDOF is distinguished from these examples by the facts that the source ${ }_{65}$ polymer is virtually achiral, that it has no photochromic groups and that it is not a specially designed polymer dedicated to CPL experiments. Induction of preferred-handed helix by CPL presented here may be extended to a wide variety of main-chain conjugated polymers.

70 We thank Dr. T. Nakanishi and Prof. Y. Hasegawa for XRD studies and Dr. Y. Tsuboi (Hokkadio Univ.) for light power measurements. This study was supported in part by the MEXT through Grants-in-Aid No. 21655037, No. 22350047, and No. 23655092 and in part by the Asahi Glass Foundation.

\section{${ }_{75}$ Notes and references}

1 Y. Okamoto and K. Hatada, J. Liq. Chromatogr., 1986, 9, 369-384.

2 T. Nakano, J. Chromatogr., 2001, A 906, 205-225.

3 Y. Okamoto and T. Nakano, Chem. Rev., 1994, 94, 349-372.

4 T. Nakano, Y. Okamoto, Chem. Rev., 2001, 101, 4013-4038.

805 E. Yashima, K. Maeda, H. Iida, Y. Furusho and K. Nagai, Chem. Rev., 2009, 109, 6102-6211.

6 J. A. Le Bell, Bull. Soc. Chim. Fr., 1874, 22, 337- 347.

7 J. H. Van't Hoff, Pamphlet, Utrecht, September 3, 1874.

8 (a) A. Moradpour, J. F. Nicoud, G. Balavoine, H. Kagan, J. Am. 85 Chem. Soc., 1971, 93, 2353-2354; (b) W. J. Bernstein, M. Calvin, O. Buchardt, J. Am. Chem. Soc., 1972, 94, 494-498.

9 (a) Y. Inoue, Chem. Rev., 1992, 92, 741-770; (b) B. L. Feringa and R. A. Delden, Angew. Chem. Int. Ed., 1999, 38, 3418-3438.

10 M. T. Bernius, M. Inbasekaran, J. O'Brien and W. Wu, Adv. Mater., $90 \quad 2000,12,1737-1750$.

11 U. Scherf and E. List, Adv. Mater., 2000, 14, 477-487.

12 M. Grell, D. D. C. Bradley, G. Ungar, J. Hill and K. S. Whitehead, Macromolecules, 1999, 32, 5810-5817.

13 (a) M. Oda, H.-G. Nothofer, G. Lieser, U. Scherf, S. C. J. Meskers 95 and D. Neher, Adv. Mater., 2000, 12, 362-365; (b) M. Oda, H.-G. Nothofer, U. Scherf, V. Sunjic, D. Richter, W. Regenstein and D. Neher, Macromolecules, 2002, 35, 6792-6798.

14 M. R. Craig, P. Jonkheijm, S. C., J. Meskers, A. P. J. J. Schenning and E. W. Meijer, Adv. Mater., 2003, 15, 1435-1438.

10015 (a) T. Nakano and T. Yade, J. Am. Chem. Soc., J. Am. Chem. Soc., 2003, 125, 15475-15484; (b) T. Nakano, K. Takewaki, T. Yade and Y. Okamoto, J. Am. Chem. Soc., 2001, 123, 9182-9183.

16 A. Mishra, R. K. Behera, P. K. Behera, B. K. Mishra, G. B. Behera, Chem. Rev., 2000, 100, 1973-2011.

10517 (a) E. J. W. List and R Guentner, Adv. Mater., 2002, 14, 374-378; (b) C. Chi, C. Im, V. Enkelmann, A. Ziegler, G. Lieser and G. Wegner, Chem. Eur. J., 2005, 11, 6833-6845.

18 A. Imamura and R. Hoffmann, J. Am. Chem. Soc., 1968, 90, 53795385.

11019 W. Kuhn, Trans. Faraday Soc., 1930, 26, 293-308.

20 (a) T. Kawasaki, M. Sato, S. Ishiguro, T. Saito, Y. Morishita, I. Sato, H. Nishino, Y. Inoue and K. Soai, J. Am. Chem. Soc., 2005, 127, 3274-3275; (b) K. Soai, T. Shibata, H. Morioka, K. Choji, Nature, 1995, 378, 767-768.

11521 M. Grell, D. D. C. Bradley, M. Inhasekarun and E. P. Woo, Adv. Mater., 1997, 9, 798-802.

22 J. Li, G. B. Schuster, K.-S. Cheon, M. M. Green and J. V. Selinger, J. Am. Chem. Soc., 2000, 122, 2603-2612.

23 (a) L. Nikolova, T. Todorov, M. Ivanov, F. Andruzzi, S. Hvilsted, P. 120 S. Ramanujam, Opt. Mater., 1997, 8, 255-258 ; (b) G. Iftime, F. L. Labarthet, A. Natansohn and P. Rochon, J. Am. Chem. Soc., 2000, 122, 12646-12650; (c) A. Natansohn and P. Rochon, Adv. Mater. 1999, $11,1387-1391$. 